\title{
Targeted long-term mental health services in Wuhan dealing with COVID-19
}

\author{
Lijun Kang $^{1} \cdot$ Gaohua Wang ${ }^{1} \cdot$ Zhongchun Liu $^{1}$
}

Received: 10 June 2020 / Accepted: 22 July 2020 / Published online: 27 July 2020

c) Springer-Verlag GmbH Germany, part of Springer Nature 2020

Since the novel coronavirus disease (COVID-19) was reported and rapidly spread in Wuhan, China, various rapid actions were taken to prevent the spread of the virus [1]. The number of confirmed cases dropped significantly, and regular commercial trade has been resuming [1]. As the pandemic has receded, the public's mental health problems have gradually become prominent.

During the COVID-19 pandemic, medical resources were directed towards the prevention and treatment of infectious diseases, and mental disease patients may not have received adequate care and timely treatment, leading to more severe symptoms. The symptoms associated with COVID-19 are also worsening, such an increased number of hand washings for patients with obsessive-compulsive disorder [2]. The uncertainties brought about by COVID-19 and pandemic prevention measures, such as isolation, wearing masks and maintaining social distance, are likely to cause serious mental health problems, such as anxiety, depression, worry, helplessness, fear, guilt, self-injury, suicide, impulse injury, and drinking, especially in infected patients, people who have close contact with infected patients, frontline medical staff, children and the elderly [2,3].

As the pandemic has gradually subsided, people have begun to resume normal life in Wuhan [1]. Although the pandemic increased concerns about health and about a sense of connectedness, psychological distress still exists. The adverse events that occurred during the epidemic, such as the pain of bereavement, economic loss, domestic violence and psychological distance caused by isolation, still exist in the memory of the public [2]. Coupled with concerns about the second-wave scenarios of the COVID-19 pandemic, the psychological difficulties of the public have become complicated [4]. Solving these problems in a timely manner may

Zhongchun Liu

zcliu6@whu.edu.cn

1 Department of Psychiatry, Renmin Hospital of Wuhan University, Wuhan 430060, China reduce the long-term mental distress created by the COVID19 pandemic [3].

Moreover, some people are at higher risk of mental illnesses in pathophysiology than they were before the pandemic. Treatment with anti-infective agents and, in particular, hospitalization have been associated with increased risks of schizophrenia and affective disorders [5]. Maternal infections have been associated with an increased risk of mental disease in offspring [6]. For some people, the negative consequences of stimulation caused by pressure activate the sympathetic adrenal medulla system, which causes mental health consequences [7]. Dynamic observation of and comprehensive support for these potentially high-risk populations are vital for alleviating the occurrence of mental diseases.

A random questionnaire survey (collected from April 19, 2020, to May 15, 2020, approved by the Clinical Research Ethics Committee of Renmin Hospital of Wuhan University) of 1140 Wuhan medical staff through the online platform Wenjuanxing found that 516 respondents (45.3\%) had depression symptoms (total score of the Patient Health Questionnaire-9 more than 4), 377 (33.1\%) had anxiety symptoms (overall score of the Generalized Anxiety Disorder-7 more than 4), 326 (28.6\%) had insomnia symptoms (total score of the Insomnia Severity Index more than 7), and $671(58.9 \%)$ had distress symptoms (overall score of the 22-item Impact of Event Scale-Revised more than 8). The results highlight that the severity of negative emotions has persisted after the pandemic. Compared with the beginning of the epidemic, the proportion of medical personnel with depression, anxiety, insomnia, and distress was 50.4\%, $44.6 \%, 34.0 \%$, and $71.5 \%$, respectively, and the proportion of mental health disturbances declined but still accounted for a large proportion [8]. Moreover, mental health disturbances such as substance dependence and suicide may affect people who have struggled against COVID-19 for a long time [2]. There is an urgent need to provide adequate and long-term mental health services to address this trauma. 
To solve these problems, during the pandemic, the government has made efforts to provide adequate protective equipment, to produce medical supplies for every patient's benefit, to advocate for positive psychological support from society as a whole, and to ensure adequate daily amounts of necessities [1]. A national psychological medical team consisting of 422 people works in Wuhan to provide mental health services, including mental health services and psychological knowledge training for both medical workers and patients. The Medical Staff Psychological Service Office was built for medical staff to relieve work stress and fatigue in a comfortable and warm environment. Furthermore, a network platform was constructed to provide online mental health assessment and intervention to support mental health needs during isolation [9].

As available medical resources and time are currently more abundant, adequate mental health services can increasingly be provided. The National Health Commission of China recently issued relevant guidelines to provide appropriate mental health services to address the risk of psychiatric morbidities for different populations, and a communitybased mental health service system is being established [10]. The psychological service network is being expanded and online serviceability is being optimized to improve the availability of psychological services. Furthermore, professional psychological service institutions and psychological service stations have been established in primary medical institutions and community service centers, which are composed of psychiatrists, psychotherapists, family doctors, and trained volunteers. Primary medical institutions provide alternative free psychological counselling services to residents. They use a mental illness self-assessment screening scale to evaluate the mental health status of residents. Patients with moderate or above abnormalities or those with emergency situations such as self-injury or impulses are referred to a psychiatric hospital for standardized diagnosis and treatment with the consent of the person or the guardian. Other residents can participate in a variety of free public services, including mental health pamphlets, community-based group rehabilitation activities, regular psychological assessment, a psychological service hotline, online self-help, and pro bono psychological services. It is necessary to provide regular mental health education to vulnerable groups in places such as schools, nursing homes, and prisons, to continue the role of the network platform and to strengthen the management of online service norms while expanding the numbers of professional online registered psychotherapists and psychiatrists. Considering the aggregated infection in closed psychiatric clinics during the pandemic and the uncertainty of COVID-19, it is also necessary to improve novel coronavirus screening of hospitalized patients and improve the prevention and management of infectious diseases in psychiatric hospitals.
Mental health workers in Wuhan have made many efforts to address this pandemic, but long-term services are still needed. This work cannot be completed in a month or 2 months; it will take years to ease the distress of the pandemic. This is not just the work of one department; it requires the attention and participation of the whole society. In the next step, people's awareness of mental health, the rational allocation and integration of resources, and the professional service ability of the community should be continuously strengthened. The construction of a psychological crisis system for public mental health and a psychological rescue system should be strengthened to ensure rapid response when a crisis occurs. Long-term mental health services should be efficiently and qualitatively recognized and supplied around the world to protect human rights and dignity.

Author contributions Writing —original draft preparation: ZL and LK; writing - review and editing: ZL, GW, and LK.

Funding This work was funded by the National Key R\&D Program of China, Grant no. 2018YFC1314600.

Availability of data and material No additional data available.

\section{Compliance with ethical standards}

Conflict of interest The authors declare no conflict of interest.

Ethics approval This study was approved by the Clinical Research Ethics Committee of Renmin Hospital of Wuhan University (WDRY2020K004).

Informed consent All participants informed consent to participate in the survey.

\section{References}

1. The State Council Information Office of the People's Republic of China (2020) China's actions to fight against the epidemic of COVID-19. https://www.scio.gov.cn/ztk/dtzt/42313/43142/index .htm. Accessed 9 Jun 2020

2. Holmes Emily A, O'Connor Rory C, Perry VH et al (2020) Multidisciplinary research priorities for the COVID-19 pandemic: a call for action for mental health science. Lancet Psychiatry 7:547-560

3. Kang L, Ma S, Chen M et al (2020) Impact on mental health and perceptions of psychological care among medical and nursing staff in Wuhan during the 2019 novel coronavirus disease outbreak: a cross-sectional study. Brain Behav Immun 87:11-17

4. López L, Rodó X (2020) The end of social confinement and COVID-19 re-emergence risk. Nature Human Behav. https://doi. org/10.1038/s41562-020-0908-8

5. Köhler O, Petersen L, Mors O et al (2017) Infections and exposure to anti-infective agents and the risk of severe mental disorders: a nationwide study. Acta Psychiatr Scand 135:97-105

6. Lydholm Cecilie N, Köhler-Forsberg O, Nordentoft M et al (2019) Parental infections before, during, and after pregnancy as risk 
factors for mental disorders in childhood and adolescence: a nationwide Danish study. Biol Psychiat 85:317-325

7. Turner AI, Smyth N, Hall SJ et al (2020) Psychological stress reactivity and future health and disease outcomes: a systematic review of prospective evidence. Psychoneuroendocrinology 114:104599

8. Lai J, Ma S, Wang Y et al (2020) Factors associated with mental health outcomes among health care workers exposed to coronavirus disease 2019. JAMA Netw Open 3(3):e203976
9. Kang L, Li Y, Hu S et al (2020) The mental health of medical workers in Wuhan, China dealing with the 2019 novel coronavirus. Lancet Psychiatry 7(3):e14

10. National Health Commission of the People's Republic of China (2020) Notice on the issuance of the new psychological pneumonia epidemic psychological counseling work plan. https://www. nhc.gov.cn/xcs/zhengcwj/202003/0beb22634f8a4a48aecf405c2 $89 \mathrm{fc} 25 \mathrm{e}$.shtml. Accessed 9 Jun 2020 